\title{
Modelling the Term Structure of Hong Kong Inter-Bank Offered Rates (HIBOR)
}

\author{
Sandy Chau, Andy Tai, Wilson Kwan \\ The Hong Kong Polytechnic University, Hong Kong, China
}

\begin{abstract}
Different single-factor models are used to estimate the term structure of Hong Kong Inter-Bank Offered Rates (HIBOR). These models use stochastic differential equations which effectively reflect market characteristics of short- and long-term interest rates, such as capability of mean reversion and interest rate level fluctuation. For the period from 2005 to early 2007, the economy of Hong Kong had been relatively stable with pretty low volatilities in interest rate. However, starting from 2008 to beginning of 2012, the Hong Kong and the world economies had been steering from relatively stable to fluctuations, the 2008 financial tsunami initiated by the U.S. had been causing financial instability globally. With the U.S. government taking quantitative easing monetary policy, U.S. interest rates fluctuated and submerged rapidly. Volatility of HIBOR was extremely sensitive to fluctuation of U.S. interest rates, since Hong Kong dollar exchange rate has been pegged with U.S. dollar. In short, during the period of early 2008 to early 2012, volatility of short-term interest rate was extremely sensitive. Obviously, the term structure of interest rate for these two periods had made major shift, combining the two periods would lead to unfavorable econometric results.
\end{abstract}

Keywords: Hong Kong Inter-Bank Offered Rates (HIBOR), dynamic interest rate term structure models, short-term interest rate volatility

\section{Introduction}

The term structure of Hong Kong Inter-Bank Offered Rates (HIBOR) is estimated by single-factor models in which dynamic process of economic variables is used as models assumption under normal circumstances in this study. These dynamic interest rate models explain the fluctuation level of interest rate term structure and enable effectively control of the interest rate risk. These models may be applied to assign appropriate pricing to interest rate related products and their derivatives. There are two types of model on term structure of interest rate. The first is equilibrium models built on the basis of "liquidity preference hypothesis theory", while the second is built upon the "expectation hypothesis theory". Both of these two models employ single stochastic differential equation to effectively reflect market characteristics of short- and long-term interest rates, such as capability of mean reversion and interest rate level fluctuation.

Sandy Chau, Ph.D., senior lecturer, School of Professional Education and Executive Development, The Hong Kong Polytechnic University, Hong Kong, China.

Andy Tai, Ph.D., senior lecturer, Hong Kong Community College, The Hong Kong Polytechnic University, Hong Kong, China.

Wilson Kwan, Ph.D., lecturer, Hong Kong Community College, The Hong Kong Polytechnic University, Hong Kong, China.

Correspondence concerning this article should be addressed to Sandy Chau, Room 807, 8/F. 8 Hung Lok Road, Hung Hom, Kowloon, Hong Kong, China. 
Short-term risk-free interest rate is an important variable in the finance sector. Upon making initial assumption on the dynamic process of short-term interest rate, a model on term structure of interest rate can be defined and eventually bond price can be deduced. During the last few decades, there had been many scholars proposing different stochastic processes to describe dynamic behavior of interest rate with restriction on different parameters estimation. In this study, different models are compared to optimize the best estimates for the term structure of interest rates for HIBOR. The models are chosen based on the dynamic process of interest rate which has the feature of non-linear mean reverting and the sensitivity of interest rate volatility to the interest rate level. A typical stochastic differential equation for short-term interest rate is stated as,

$$
d r(t)=\mu\left(r_{t}\right) d t+\eta\left(r_{t}\right) d W_{t}
$$

where $\mu\left(r_{t}\right)$ is a drift function and $\eta\left(r_{t}\right)$ is a diffusion function. This equation can be extended by putting $\mu\left(r_{t}\right)=$ $\left(\alpha+\beta r_{t}\right)$ and $\eta\left(r_{t}\right)=\sigma r_{t}^{\gamma}$ and rewritten as:

$$
d r_{t}=\left(\alpha+\beta r_{t}\right) d t+\sigma r_{t}^{\gamma} d W_{t}
$$

where $r_{t}$ is short-term interest rate and $d W$ is the increasing rate of Wiener process which is in compliance with the standard Brownian movement.

Theoretically, short-term interest rate would revert to the mean value of long-term interest rate, otherwise the variance of short-term interest rate would endlessly increase to infinity, therefore short-term interest rates have characteristic of mean reversion. In such case, the value of $\beta$ should be negative while the value of $\alpha$ should be positive.

Volatility of short-term rate is a function of short-term interest rate level and $\gamma$ is the sensitivity coefficient of short-term interest rate volatility to short-term interest rate level. Therefore $\gamma>1$ characterizes short rate volatility which is over-sensitive to interest rate level, while in contrary $\gamma<1$ means that volatility is not sensitive to interest rate level, and $\sigma$ would not equal to zero. $\sigma r_{t}^{\gamma}$ in equation (2) represents the volatility of interest rate and $\gamma$ represents sensitivity coefficient of short-term interest rate volatility to short-term interest rate level.

In the past, different models with different restrictions to $\alpha, \beta, \sigma$, and $\gamma$ were used to established short-term interest rate models. Merton was the first to propose a relatively simple single-factor equilibrium model in 1973, his model is equipped with a stochastic drift component and a diffusion component of risk-free short-term interest rate, to calculate pricing for discount bonds; accordingly his work eventually won him the Nobel Memorial Prize in economic science in 1997. However, Merton's model (1973) suffered from the limitations that interest rate can be negative and that the mean reversion characteristic of market interest rate is violated when the value of $\mu$ is zero.

The second model is the famous geometric Brownian motion (GBM) model developed by Black and Scholes in 1973: It assumes that interest rate is log-normally distributed and follows geometric Brownian movement, the model is best known and is the most widely used option pricing model in financial markets.

The third model by Cox and Ross in 1976 is the CEV model, which is also known as the constant elasticity of variance model. Having the variance of interest rate at $2 \gamma$ which is independent of interest rate, it is therefore characterized as a constant elasticity of variance process.

The fourth model being developed by Vasicek in 1977 is an interest rate equation with significant mean reversion characteristic; it is in fact the Metron model (1973) if the equation (2) is restricted with $\beta=0$; 
Vasicek (1977) can only overcome the mean reversion concern as seen in the Metron model (1973), but it still fails to address the assumption of non-negative interest rate by allowing short-term interest rate to be negative.

The fifth model is a single factor continuous time equilibrium model proposed by Dothan in 1978, the model calculates discount bond price by assuming stochastic short-term interest rate follows a geometric Wiener process, especially with a zero drift component indicating that interest rate is random walk by itself thus denying the growth characteristics of interest rate.

The sixth model is the term structure of interest rate model built by Brennan and Schwartz in 1979 to calculate swap pricing; it also strictly limits the interest rate to be non-negative.

The CIR VR model built by Cox, Ingersoll, and Ross (1985) is the seventh model as shown. It is also known as variable-rate term structure of interest rate model applying to variable-rate securities.

The eighth model introduced by Cox, Ingersoll, and Ross in 1985 is known as the CIR SR model. It is the famous term structure of interest rate model being developed from the general equilibrium models basing on the platform of continuous time series; the model assumes short-term interest rate follows a square root process which leads to only positive interest rate; it overcomes the Vasicek's concern (1977) on negative rate, while maintaining the property of mean reversion.

The ninth model is proposed by Chan, Karolyi, Longstaff, and Sanders (1992), known as CKLS model, with no restrictions on estimation of $\alpha, \beta, \sigma$, and $\gamma$.

Table 1

Dynamic Interest Rate Term Structure Models of Different Era, and Their Corresponding Restrictions on Parameters

\begin{tabular}{lllll}
\hline Model & $\alpha$ & $\beta$ & $\sigma$ & $\gamma$ \\
\hline 1. Merton (1973) & $\mathrm{NR}$ & 0 & $\mathrm{NR}$ & 0 \\
2. Black and Scholes (1973), GBM & 0 & $\mathrm{NR}$ & $\mathrm{NR}$ & 1 \\
3. Cox and Ross (1976), CEV & 0 & $\mathrm{NR}$ & $\mathrm{NR}$ & $\mathrm{NR}$ \\
4. Vasicek (1977) & $\mathrm{NR}$ & $\mathrm{NR}$ & $\mathrm{NR}$ & 0 \\
5. Dothan (1978) & 0 & 0 & $\mathrm{NR}$ & 1 \\
6. Brennan-Schwartz (1979) & $\mathrm{NR}$ & $\mathrm{NR}$ & $\mathrm{NR}$ & 1 \\
7. Cox, Ingersoll, and Ross (1985), CIR VR & 0 & 0 & $\mathrm{NR}$ & 1.5 \\
8. Cox, Ingersoll, and Ross (1985), CIR SR & $\mathrm{NR}$ & $\mathrm{NR}$ & $\mathrm{NR}$ & 0.5 \\
9. Chan et al. (1992) & $\mathrm{NR}$ & $\mathrm{NR}$ & $\mathrm{NR}$ & $\mathrm{NR}$ \\
\hline
\end{tabular}

Note. $\mathrm{NR}=$ No restriction.

Past research (Chan et al., 1992; Nowman, 1997) indicated that sensitivity of short-term interest rate volatility to short-term interest rate level was having coefficient $\gamma$ equaled 1.50 and 1.36 respectively. The value of $\gamma>1$ is interpreted as the volatility is over-sensitive to the short-term interest rate level. Tse (1995), Dahlquist (1996), Hiraki and Takezawa (1997), Manus and Watt (1999), and Corradi and Swanson (2011) estimated different short-term interest rate models under the CKLS framework, using data of different countries and their results indicated that sensitivity of short-term interest rate volatility to short-term interest rate level is having coefficient $\gamma$ ranging from 0.21 to 0.72 . The value of $\gamma<1$ is interpreted as low sensitivity of volatility to the short-term interest rate level. Nowman (1998), Manus and Watt (1999), and Duong and Swanson (2011) estimated short-term interest rate models under the CKLS framework to examine over 10 countries and their results indicated that sensitivity of short-term interest rate volatility to short-term interest rate level is having 
coefficient $\gamma$ ranging from 0.1 to 2.8 , both $\gamma<1$ and $\gamma>1$ appeared statistically significant at $5 \%$ level in different regions, meaning that the sensitivity can exist from low to high level.

However, it is noted that some financial crises or extreme policies impacted the interest rate volatility, for example, Bliss and Smith (1994) discovered that the relationship between volatility of short rate and short-term interest rate level depended upon whether the interest rate data included the wall street stock market crash period during Oct. 1987; the volatility was low if these data were excluded, but volatility is high when these data were included. Cai and Swanson (2011) also found similar results on the European short-term interest rate. Therefore, inclusion of interest rate during financial crisis might cause inaccurate coefficient estimations and incorrect statistical significance in the term structure model.

\section{Methodology}

To measure the sensitivity of short-term interest rate volatility to short-term interest rate level, the maximum likelihood estimation (MLE) by Nowman (1997) is adopted and the estimation function is

$$
\mathrm{MLF}=\frac{1}{2} \sum_{t=1}^{n}\left[\ln \left(2 \pi \sigma^{2} r_{t}^{2 \gamma} d t\right)+\left(\frac{d r_{t}-\left(\alpha+\beta r_{t}\right)}{\sqrt{\sigma^{2} r_{t}^{2 \gamma} d t}}\right)^{2}\right]
$$

where $n$ is the number of data sample. The 1-month, 3-month, 6-month, 9-month, and 12-month HIBOR were sourced from datastream of Thomson Reuter. Data are daily closing rates for the period from 3rd Jan., 2005 to 22nd Feb., 2012. In this research, there are a total of 1,863 data samples.

For comparison, different restrictions to $\alpha, \beta, \sigma$, and $\gamma$ are applied based on models by previous work to establish different interest rate term structure models as stated in Table 2.

Table 2

Estimates of Parameter Vector $\alpha, \beta$, $\sigma$, and $\gamma$ for Different Models (for Period 1)

\begin{tabular}{|c|c|c|c|c|c|c|}
\hline Model & Term & $\alpha$ & $\beta$ & $\sigma$ & $\gamma$ & LLF \\
\hline \multirow{10}{*}{ 1. Merton (1973) } & 1-month & 0.9757 & 0.0000 & 1.6684 & 0.0000 & 650.4692 \\
\hline & & $(4.7633)$ & $(1.0000)$ & (2.0234) & $(1.0000)$ & \\
\hline & 3-month & 1.0110 & 0.0000 & -1.1350 & 0.0000 & 950.9272 \\
\hline & & $(6.2692)$ & $(1.0000)$ & (1.3697) & $(1.0000)$ & \\
\hline & 6-month & 0.9710 & 0.0000 & 0.9842 & 0.0000 & $1,062.1090$ \\
\hline & & $(4.3587)$ & $(1.0000)$ & (1.1400) & $(1.0000)$ & \\
\hline & 9-month & 0.8885 & 0.0000 & -0.9231 & 0.0000 & $1,112.0827$ \\
\hline & & $(4.3652)$ & $(1.0000)$ & (1.1070) & (1.0000) & \\
\hline & 12-month & 0.8128 & 0.0000 & -0.9087 & 0.0000 & $1,124.3541$ \\
\hline & & $(4.6015)$ & $(1.0000)$ & (1.1504) & $(1.0000)$ & \\
\hline \multirow{10}{*}{$\begin{array}{l}\text { 2. Black and Scholes (1973), } \\
\text { GBM }\end{array}$} & 1-month & 0.0000 & 1.1141 & -0.7596 & 1.0000 & 277.7238 \\
\hline & & $(1.0000)$ & $(4.8175)$ & $(0.1003)$ & $(1.0000)$ & \\
\hline & 3-month & 0.0000 & 0.9005 & -0.5379 & 1.0000 & 511.2083 \\
\hline & & $(1.0000)$ & $(2.4174)$ & $(0.0680)$ & $(1.0000)$ & \\
\hline & 6-month & 0.0000 & 0.6931 & 0.4204 & 1.0000 & 674.8548 \\
\hline & & (1.0000) & (1.2075) & $(0.1690)$ & (1.0000) & \\
\hline & 9-month & 0.0000 & 0.5600 & -0.3581 & 1.0000 & 781.1243 \\
\hline & & $(1.0000)$ & (1.3943) & $(0.1614)$ & $(1.0000)$ & \\
\hline & 12-month & 0.0000 & 0.4712 & -0.3219 & 1.0000 & 849.2370 \\
\hline & & $(1.0000)$ & $(2.2325)$ & $(0.2344)$ & $(1.0000)$ & \\
\hline
\end{tabular}


Table 2 continued

\begin{tabular}{|c|c|c|c|c|c|c|}
\hline Model & Term & $\alpha$ & $\beta$ & $\sigma$ & $\gamma$ & LLF \\
\hline \multirow{5}{*}{ 3. Cox and Ross (1976), CEV } & 1-month & $\begin{array}{l}0.0000 \\
(1.0000)\end{array}$ & $\begin{array}{l}-0.0247 \\
(0.3367)\end{array}$ & $\begin{array}{l}2.0342 \\
(0.1702)\end{array}$ & $\begin{array}{l}-0.1596 \\
(0.0638)\end{array}$ & 653.2505 \\
\hline & 3-month & $\begin{array}{l}0.0000 \\
(1.0000)\end{array}$ & $\begin{array}{l}0.0159 \\
(0.2739)\end{array}$ & $\begin{array}{l}1.7799 \\
(0.1688)\end{array}$ & $\begin{array}{l}-0.3581 \\
(0.0697)\end{array}$ & 966.0136 \\
\hline & 6-month & $\begin{array}{l}0.0000 \\
(1.0000)\end{array}$ & $\begin{array}{l}0.0128 \\
(0.2642)\end{array}$ & $\begin{array}{l}2.0023 \\
(0.2129)\end{array}$ & $\begin{array}{l}-0.5562 \\
(0.0765)\end{array}$ & $1,092.6061$ \\
\hline & 9-month & $\begin{array}{l}0.0000 \\
(1.0000)\end{array}$ & $\begin{array}{l}0.0010 \\
(0.2533)\end{array}$ & $\begin{array}{l}2.2372 \\
(0.3364)\end{array}$ & $\begin{array}{l}-0.7276 \\
(0.1029)\end{array}$ & $1,154.0921$ \\
\hline & 12-month & $\begin{array}{l}0.0000 \\
(1.0000)\end{array}$ & $\begin{array}{l}-0.0068 \\
(0.2458) \\
\end{array}$ & $\begin{array}{l}2.6474 \\
(0.3610) \\
\end{array}$ & $\begin{array}{l}-0.8075 \\
(0.0945) \\
\end{array}$ & $1,166.2051$ \\
\hline \multirow{5}{*}{ 4. Vasicek (1977) } & 1-month & $\begin{array}{l}14.6426 \\
(38.9047)\end{array}$ & $\begin{array}{l}-3.6179 \\
(10.4430)\end{array}$ & $\begin{array}{l}1.6538 \\
(1.9457)\end{array}$ & $\begin{array}{l}0.0000 \\
(1.0000)\end{array}$ & 657.3259 \\
\hline & 3-month & $\begin{array}{l}13.5126 \\
(4.7241)\end{array}$ & $\begin{array}{l}-3.2095 \\
(1.1523)\end{array}$ & $\begin{array}{l}-1.1199 \\
(1.2823)\end{array}$ & $\begin{array}{l}0.0000 \\
(1.0000)\end{array}$ & 961.3882 \\
\hline & 6-month & $\begin{array}{l}13.7422 \\
(9.9615)\end{array}$ & $\begin{array}{l}-3.1953 \\
(1.2779)\end{array}$ & $\begin{array}{l}-0.9687 \\
(1.1129)\end{array}$ & $\begin{array}{l}0.0000 \\
(1.0000)\end{array}$ & $1,074.4813$ \\
\hline & 9-month & $\begin{array}{l}13.7477 \\
(21.0215)\end{array}$ & $\begin{array}{l}-3.1613 \\
(4.7310)\end{array}$ & $\begin{array}{l}-0.9084 \\
(1.1156)\end{array}$ & $\begin{array}{l}0.0000 \\
(1.0000)\end{array}$ & $1,124.6491$ \\
\hline & 12-month & $\begin{array}{l}13.8326 \\
(6.0870)\end{array}$ & $\begin{array}{l}-3.1547 \\
(1.4330)\end{array}$ & $\begin{array}{l}-0.8950 \\
(1.1440)\end{array}$ & $\begin{array}{l}0.0000 \\
(1.0000)\end{array}$ & $1,136.2535$ \\
\hline \multirow{5}{*}{ 5. Dothan (1978) } & 1-month & $\begin{array}{l}0.0000 \\
(1.0000)\end{array}$ & $\begin{array}{l}0.0000 \\
(1.0000)\end{array}$ & $\begin{array}{l}-0.7628 \\
(0.0751)\end{array}$ & $\begin{array}{l}1.0000 \\
(1.0000)\end{array}$ & 274.4080 \\
\hline & 3-month & $\begin{array}{l}0.0000 \\
(1.0000)\end{array}$ & $\begin{array}{l}0.0000 \\
(1.0000)\end{array}$ & $\begin{array}{l}-0.5409 \\
(0.0649)\end{array}$ & $\begin{array}{l}1.0000 \\
(1.0000)\end{array}$ & 506.8949 \\
\hline & 6-month & $\begin{array}{l}0.0000 \\
(1.0000)\end{array}$ & $\begin{array}{l}0.0000 \\
(1.0000)\end{array}$ & $\begin{array}{l}0.4227 \\
(0.1786)\end{array}$ & $\begin{array}{l}1.0000 \\
(1.0000)\end{array}$ & 670.6719 \\
\hline & 9-month & $\begin{array}{l}0.0000 \\
(1.0000)\end{array}$ & $\begin{array}{l}0.0000 \\
(1.0000)\end{array}$ & $\begin{array}{l}-0.3598 \\
(0.2303)\end{array}$ & $\begin{array}{l}1.0000 \\
(1.0000)\end{array}$ & 777.3571 \\
\hline & 12-month & $\begin{array}{l}0.0000 \\
(1.0000)\end{array}$ & $\begin{array}{l}0.0000 \\
(1.0000)\end{array}$ & $\begin{array}{l}-0.3232 \\
(0.2583)\end{array}$ & $\begin{array}{l}1.0000 \\
(1.0000)\end{array}$ & 845.9330 \\
\hline \multirow{5}{*}{$\begin{array}{l}\text { 6. Brennan and } \\
(1979)\end{array}$} & 1-month & $\begin{array}{l}11.3958 \\
(5.3663)\end{array}$ & $\begin{array}{l}-2.7053 \\
(2.2348)\end{array}$ & $\begin{array}{l}-0.7123 \\
(0.1752)\end{array}$ & $\begin{array}{l}1.0000 \\
(1.0000)\end{array}$ & 327.8966 \\
\hline & 3-month & $\begin{array}{l}17.2750 \\
(5.5264)\end{array}$ & $\begin{array}{l}-4.2924 \\
(1.8846)\end{array}$ & $\begin{array}{l}0.4747 \\
(0.1967)\end{array}$ & $\begin{array}{l}1.0000 \\
(1.0000)\end{array}$ & 608.6652 \\
\hline & 6-month & $\begin{array}{l}18.0878 \\
(6.9990)\end{array}$ & $\begin{array}{l}-4.3627 \\
(2.0899)\end{array}$ & $\begin{array}{l}0.3808 \\
(0.2318)\end{array}$ & $\begin{array}{l}1.0000 \\
(1.0000)\end{array}$ & 752.1396 \\
\hline & 9-month & $\begin{array}{l}17.9845 \\
(8.0734)\end{array}$ & $\begin{array}{l}-4.2571 \\
(2.1093)\end{array}$ & $\begin{array}{l}-0.3309 \\
(0.2495)\end{array}$ & $\begin{array}{l}1.0000 \\
(1.0000)\end{array}$ & 842.6361 \\
\hline & 12-month & $\begin{array}{l}18.3053 \\
(8.7539)\end{array}$ & $\begin{array}{l}-4.2824 \\
(2.3488)\end{array}$ & $\begin{array}{l}-0.3011 \\
(0.2480)\end{array}$ & $\begin{array}{l}1.0000 \\
(1.0000)\end{array}$ & 901.2840 \\
\hline \multirow{5}{*}{ 7. Cox et al. (1985), CIR VR } & 1-month & $\begin{array}{l}0.0000 \\
(1.0000)\end{array}$ & $\begin{array}{l}0.0000 \\
(1.0000)\end{array}$ & $\begin{array}{l}-0.8785 \\
(0.5161)\end{array}$ & $\begin{array}{l}1.5000 \\
(1.0000)\end{array}$ & -328.9352 \\
\hline & 3-month & $\begin{array}{l}0.0000 \\
(1.0000)\end{array}$ & $\begin{array}{l}0.0000 \\
(1.0000)\end{array}$ & $\begin{array}{l}0.5985 \\
(0.2808)\end{array}$ & $\begin{array}{l}1.5000 \\
(1.0000)\end{array}$ & -83.1113 \\
\hline & 6-month & $\begin{array}{l}0.0000 \\
(1.0000)\end{array}$ & $\begin{array}{l}0.0000 \\
(1.0000)\end{array}$ & $\begin{array}{l}0.3812 \\
(0.0154)\end{array}$ & $\begin{array}{l}1.5000 \\
(1.0000)\end{array}$ & 225.9116 \\
\hline & 9-month & $\begin{array}{l}0.0000 \\
(1.0000)\end{array}$ & $\begin{array}{l}0.0000 \\
(1.0000)\end{array}$ & $\begin{array}{l}-0.2833 \\
(0.0942)\end{array}$ & $\begin{array}{l}1.5000 \\
(1.0000)\end{array}$ & 428.9121 \\
\hline & 12-month & $\begin{array}{l}0.0000 \\
(1.0000)\end{array}$ & $\begin{array}{l}0.0000 \\
(1.0000)\end{array}$ & $\begin{array}{l}-0.2325 \\
(0.1235)\end{array}$ & $\begin{array}{l}1.5000 \\
(1.0000)\end{array}$ & 560.5695 \\
\hline
\end{tabular}


Table 2 continued

\begin{tabular}{|c|c|c|c|c|c|c|}
\hline Model & Term & $\alpha$ & $\beta$ & $\sigma$ & $\gamma$ & LLF \\
\hline \multirow{10}{*}{ 8. Cox et al. (1985), CIR SR } & 1-month & 12.1495 & -2.9579 & 0.9595 & 0.5000 & 588.7309 \\
\hline & & (11.0919) & $(7.4841)$ & $(1.0500)$ & $(1.0000)$ & \\
\hline & 3-month & 14.6072 & -3.4905 & -0.6558 & 0.5000 & 867.7039 \\
\hline & & $(3.1409)$ & $(1.1967)$ & $(0.6281)$ & $(1.0000)$ & \\
\hline & 6-month & 15.3543 & -3.5986 & -0.5613 & 0.5000 & 974.7611 \\
\hline & & $(5.7203)$ & $(1.5468)$ & $(0.5388)$ & $(1.0000)$ & \\
\hline & 9-month & 15.5193 & -3.5968 & 0.5167 & 0.5000 & $1,029.8464$ \\
\hline & & $(17.7181)$ & $(5.2120)$ & $(0.6282)$ & $(1.0000)$ & \\
\hline & 12-month & 15.7794 & -3.6264 & -0.4954 & 0.5000 & $1,055.1591$ \\
\hline & & $(5.7954)$ & $(1.3757)$ & $(0.4381)$ & $(1.0000)$ & \\
\hline \multirow{10}{*}{ 9. Chan et al. (1992) } & 1-month & 15.4490 & -3.8210 & 1.9553 & -0.1347 & 659.5833 \\
\hline & & $(4.5742)$ & $(1.1560)$ & $(0.1697)$ & $(0.0657)$ & \\
\hline & 3-month & 13.4931 & -3.2050 & 1.6712 & -0.3170 & 973.1489 \\
\hline & & $(3.5047)$ & $(0.8545)$ & $(0.1490)$ & $(0.0658)$ & \\
\hline & 6-month & 13.0577 & -3.0317 & 1.8667 & -0.5106 & $1,099.3733$ \\
\hline & & (3.5634) & $(0.8353)$ & $(0.2109)$ & $(0.0812)$ & \\
\hline & 9-month & 12.3203 & -2.8254 & 2.2076 & -0.6805 & $1,159.8577$ \\
\hline & & (3.6177) & $(0.8356)$ & $(0.2861)$ & $(0.0921)$ & \\
\hline & 12-month & 11.9094 & -2.7074 & 2.4520 & -0.7570 & $1,171.2996$ \\
\hline & & (4.2947) & $(0.9636)$ & $(0.3551)$ & $(0.1022)$ & \\
\hline
\end{tabular}

Notes. At MLE maximization, data from daily closing rates for the period are from 3rd Jan., 2005 to 31st Dec., 2007, total 781 data sample; standard deviations are shown in parentheses.

Due to the non-satisfactory results obtained from the full period analysis, it is decided to split the time period into two sections. The first period is from 3rd Jan., 2005 to 31st Dec., 2007 and the second period covers 1st Jan., 2008 to 22nd Feb., 2012. Table 3 shows empirical test results for the first time period of 3rd Jan., 2005 to 31 st Dec., 2007, covering interest rate dynamic model one to nine.

Table 3 shows empirical test results for the second time period of 1st Jan., 2008 to 31st Dec., 2007, covering interest rate dynamic model one to nine.

Table 3

Estimates of Parameter Vector $\alpha, \beta, \sigma$, and $\gamma$ for Different Models (for Period 2)

\begin{tabular}{lllllll}
\hline Model & Term & $\alpha$ & $\beta$ & $\sigma$ & $\gamma$ & \multicolumn{2}{l}{ LLF } \\
\hline & 1-month & -0.6870 & 0.0000 & -1.7989 & 0.0000 & 820.0568 \\
& $(15.5695)$ & $(1.0000)$ & $(1.8795)$ & $(1.0000)$ & \\
& 3-month & -0.7106 & 0.0000 & 0.9036 & 0.0000 & $1,564.3015$ \\
& & $(0.9159)$ & $(1.0000)$ & $(0.8828)$ & $(1.0000)$ & \\
1. Merton (1973) & \multirow{3}{*}{ 6-month } & -0.6970 & 0.0000 & 0.7422 & 0.0000 & $1,777.0288$ \\
& & $(2.7580)$ & $(1.0000)$ & $(0.8221)$ & $(1.0000)$ & $1,751.9439$ \\
& \multirow{3}{*}{ 9-month } & -0.6794 & 0.0000 & 0.7597 & 0.0000 & $(1.0000)$ \\
& \multirow{3}{*}{ 12-month } & $(1.2036)$ & $(1.0000)$ & $(0.7229)$ & 0.0000 & $1,726.3183$ \\
& & -0.6083 & 0.0000 & 0.7779 & $(1.0000)$ & \\
\hline
\end{tabular}


Table 3 continued

\begin{tabular}{|c|c|c|c|c|c|c|}
\hline Model & Term & $\alpha$ & $\beta$ & $\sigma$ & $\gamma$ & LLF \\
\hline \multirow{8}{*}{$\begin{array}{l}\text { 2. Black and Scholes (1973), } \\
\text { GBM }\end{array}$} & 1-month & $\begin{array}{l}0.0000 \\
(1.0000)\end{array}$ & $\begin{array}{l}0.0288 \\
(5.9205)\end{array}$ & $\begin{array}{l}1.1344 \\
(0.9099)\end{array}$ & $\begin{array}{l}1.0000 \\
(1.0000)\end{array}$ & $2,646.9854$ \\
\hline & 3-month & $\begin{array}{l}0.0000 \\
(10000)\end{array}$ & $\begin{array}{l}-0.3510 \\
(33034)\end{array}$ & $\begin{array}{l}0.5508 \\
(02755)\end{array}$ & $\begin{array}{l}1.0000 \\
(10000)\end{array}$ & $2,919.5711$ \\
\hline & 6-month & 0.0000 & -0.3360 & -0.4383 & 1.0000 & $2,775.1486$ \\
\hline & & $(1.0000)$ & $(2.0406)$ & $(0.1074)$ & $(1.0000)$ & \\
\hline & 9-month & 0.0000 & -0.3346 & 0.4042 & 1.0000 & $2,619.0742$ \\
\hline & & $(1.0000)$ & $(1.5643)$ & $(0.0518)$ & $(1.0000)$ & \\
\hline & 12-month & 0.0000 & -0.2530 & 0.3871 & 1.0000 & $2,412.9352$ \\
\hline & & $(1.0000)$ & $(1.1450)$ & $(0.1266)$ & $(1.0000)$ & \\
\hline \multirow{10}{*}{ 3. Cox and Ross (1976), CEV } & 1-month & 0.0000 & 0.2744 & 1.2772 & 1.1191 & $2,676.9510$ \\
\hline & & $(1.0000)$ & $(5.3843)$ & $(0.0486)$ & $(0.0155)$ & \\
\hline & 3-month & 0.0000 & -0.2415 & 0.5858 & 1.0979 & $2,933.2483$ \\
\hline & & $(1.0000)$ & $(3.3263)$ & $(0.0220)$ & $(0.0217)$ & \\
\hline & 6-month & 0.0000 & -0.2512 & -0.4531 & 1.1099 & $2,786.5001$ \\
\hline & & $(1.0000)$ & $(0.8650)$ & $(0.0135)$ & $(0.0240)$ & \\
\hline & 9-month & 0.0000 & 2.1425 & 0.4067 & 1.2400 & $2,656.6200$ \\
\hline & & $(1.0000)$ & $(1.7344)$ & $(0.0096)$ & $(0.0289)$ & \\
\hline & 12-month & 0.0000 & -0.1291 & 0.3636 & 1.3203 & $2,459.0599$ \\
\hline & & $(1.0000)$ & $(1.1592)$ & $(0.0081)$ & $(0.0364)$ & \\
\hline \multirow{10}{*}{ 4. Vasicek (1977) } & 1-month & 1.1769 & -2.9790 & 1.7909 & 0.0000 & 824.8390 \\
\hline & & (30.1064) & $(17.8601)$ & (1.9567) & $(1.0000)$ & \\
\hline & 3-month & 0.3878 & -1.3410 & 0.9001 & 0.0000 & $1,568.5306$ \\
\hline & & $(1.8562)$ & $(1.1365)$ & (1.1211) & $(1.0000)$ & \\
\hline & 6-month & 0.4420 & -1.1529 & 0.7391 & 0.0000 & $1,781.6058$ \\
\hline & & (22.6141) & $(25.0897)$ & $(0.6881)$ & $(1.0000)$ & \\
\hline & 9-month & 0.8288 & -1.0917 & 0.7570 & 0.0000 & $1,755.6811$ \\
\hline & & $(0.7691)$ & $(0.7485)$ & $(0.7609)$ & $(1.0000)$ & \\
\hline & 12-month & 0.7691 & -1.0674 & 0.7756 & 0.0000 & $1,729.4550$ \\
\hline & & $(1.3462)$ & $(3.8512)$ & $(0.7558)$ & $(1.0000)$ & \\
\hline \multirow{10}{*}{ 5. Dothan (1978) } & 1-month & 0.0000 & 0.0000 & 1.1344 & 1.0000 & $2,646.9840$ \\
\hline & & $(1.0000)$ & $(1.0000)$ & $(0.8591)$ & $(1.0000)$ & \\
\hline & 3-month & 0.0000 & 0.0000 & 0.5512 & 1.0000 & $2,918.7005$ \\
\hline & & $(1.0000)$ & $(1.0000)$ & $(0.2712)$ & $(1.0000)$ & \\
\hline & 6-month & 0.0000 & 0.0000 & 0.4388 & 1.0000 & $2,773.8897$ \\
\hline & & $(1.0000)$ & $(1.0000)$ & $(0.0875)$ & $(1.0000)$ & \\
\hline & 9-month & 0.0000 & 0.0000 & 0.4047 & 1.0000 & $2,617.6061$ \\
\hline & & $(1.0000)$ & $(1.0000)$ & $(0.0528)$ & $(1.0000)$ & \\
\hline & 12-month & 0.0000 & 0.0000 & 0.3875 & 1.0000 & $2,412.0200$ \\
\hline & & $(1.0000)$ & $(1.0000)$ & $(0.1309)$ & $(1.0000)$ & \\
\hline \multirow{10}{*}{ 6. Brennan and Schwartz (1979) } & 1-month & 0.2661 & -1.4361 & 1.1319 & 1.0000 & $2,646.3440$ \\
\hline & & $(1.5915)$ & $(8.5693)$ & $(0.6027)$ & $(1.0000)$ & \\
\hline & 3-month & 0.2810 & -1.2363 & 0.5493 & 1.0000 & $2,922.4991$ \\
\hline & & $(0.4082)$ & $(1.6958)$ & $(0.2311)$ & $(1.0000)$ & \\
\hline & 6-month & 0.4499 & -1.2212 & 0.4370 & 1.0000 & $2,778.4426$ \\
\hline & & $(0.8725)$ & $(0.4461)$ & $(0.2879)$ & $(1.0000)$ & \\
\hline & 9-month & 0.5847 & -1.1894 & -0.4032 & 1.0000 & $2,621.7808$ \\
\hline & & $(0.2634)$ & $(0.4491)$ & $(0.0529)$ & $(1.0000)$ & \\
\hline & 12-month & 0.7905 & -1.1134 & -0.3863 & 1.0000 & $2,415.1944$ \\
\hline & & $(0.4395)$ & $(0.5296)$ & $(0.1420)$ & $(1.0000)$ & \\
\hline
\end{tabular}


Table 3 continued

\begin{tabular}{|c|c|c|c|c|c|c|}
\hline Model & Term & $\alpha$ & $\beta$ & $\sigma$ & $\gamma$ & LLF \\
\hline \multirow{5}{*}{ 7. Cox et al. (1985), CIR VR } & 1-month & $\begin{array}{l}0.0000 \\
(1.0000)\end{array}$ & $\begin{array}{l}0.0000 \\
(1.0000)\end{array}$ & $\begin{array}{l}-2.5094 \\
(5.4056)\end{array}$ & $\begin{array}{l}1.5000 \\
(1.0000)\end{array}$ & $2,452.9614$ \\
\hline & 3-month & $\begin{array}{l}0.0000 \\
(1.0000)\end{array}$ & $\begin{array}{l}0.0000 \\
(1.0000)\end{array}$ & $\begin{array}{l}0.9384 \\
(1.3992)\end{array}$ & $\begin{array}{l}1.5000 \\
(1.0000)\end{array}$ & $2,753.5353$ \\
\hline & 6-month & $\begin{array}{l}0.0000 \\
(1.0000)\end{array}$ & $\begin{array}{l}0.0000 \\
(1.0000)\end{array}$ & $\begin{array}{l}0.5886 \\
(0.5214)\end{array}$ & $\begin{array}{l}1.5000 \\
(1.0000)\end{array}$ & $2,670.9069$ \\
\hline & 9-month & $\begin{array}{l}0.0000 \\
(1.0000)\end{array}$ & $\begin{array}{l}0.0000 \\
(1.0000)\end{array}$ & $\begin{array}{l}0.4404 \\
(0.1867)\end{array}$ & $\begin{array}{l}1.5000 \\
(1.0000)\end{array}$ & $2,618.8819$ \\
\hline & 12-month & $\begin{array}{l}0.0000 \\
(1.0000) \\
\end{array}$ & $\begin{array}{l}0.0000 \\
(1.0000) \\
\end{array}$ & $\begin{array}{l}-0.3639 \\
(0.0255) \\
\end{array}$ & $\begin{array}{l}1.5000 \\
(1.0000) \\
\end{array}$ & $2,445.9985$ \\
\hline \multirow{5}{*}{ 8. Cox et al. (1985), CIR SR } & 1-month & $\begin{array}{l}0.2884 \\
(13.7837)\end{array}$ & $\begin{array}{l}-1.5586 \\
(25.8274)\end{array}$ & $\begin{array}{l}-1.1819 \\
(0.7408)\end{array}$ & $\begin{array}{l}0.5000 \\
(1.0000)\end{array}$ & $1,938.3990$ \\
\hline & 3-month & $\begin{array}{l}0.2675 \\
(1.2334)\end{array}$ & $\begin{array}{l}-1.1938 \\
(1.7371)\end{array}$ & $\begin{array}{l}-0.5885 \\
(0.3869)\end{array}$ & $\begin{array}{l}0.5000 \\
(1.0000)\end{array}$ & $2,437.9088$ \\
\hline & 6-month & $\begin{array}{l}0.3868 \\
(2.3729)\end{array}$ & $\begin{array}{l}-1.0970 \\
(2.1751)\end{array}$ & $\begin{array}{l}-0.4922 \\
(0.3861)\end{array}$ & $\begin{array}{l}0.5000 \\
(1.0000)\end{array}$ & $2,435.5481$ \\
\hline & 9-month & $\begin{array}{l}0.4811 \\
(3.1879)\end{array}$ & $\begin{array}{l}-1.0380 \\
(2.1649)\end{array}$ & $\begin{array}{l}0.4987 \\
(0.3269)\end{array}$ & $\begin{array}{l}0.5000 \\
(1.0000)\end{array}$ & $2,299.4644$ \\
\hline & 12-month & $\begin{array}{l}0.6975 \\
(1.4202) \\
\end{array}$ & $\begin{array}{l}-1.0119 \\
(1.1299) \\
\end{array}$ & $\begin{array}{l}0.5067 \\
(0.4463) \\
\end{array}$ & $\begin{array}{l}0.5000 \\
(1.0000)\end{array}$ & $2,155.9214$ \\
\hline \multirow{5}{*}{ 9. Chan et al. (1992) } & 1-month & $\begin{array}{l}0.2848 \\
(0.1065)\end{array}$ & $\begin{array}{l}-1.5829 \\
(0.9140)\end{array}$ & $\begin{array}{l}1.2762 \\
(0.0392)\end{array}$ & $\begin{array}{l}1.1208 \\
(0.0167)\end{array}$ & $2,680.1175$ \\
\hline & 3-month & $\begin{array}{l}0.2824 \\
(0.1129)\end{array}$ & $\begin{array}{l}-1.2434 \\
(0.4848)\end{array}$ & $\begin{array}{l}0.5843 \\
(0.0151)\end{array}$ & $\begin{array}{l}1.0984 \\
(0.0187)\end{array}$ & $2,936.3904$ \\
\hline & 6-month & $\begin{array}{l}0.4708 \\
(0.1762)\end{array}$ & $\begin{array}{l}-1.2727 \\
(0.4401)\end{array}$ & $\begin{array}{l}0.4518 \\
(0.0108)\end{array}$ & $\begin{array}{l}1.1110 \\
(0.0235)\end{array}$ & $2,790.0831$ \\
\hline & 9-month & $\begin{array}{l}0.6811 \\
(0.2819)\end{array}$ & $\begin{array}{l}-1.3616 \\
(0.5225)\end{array}$ & $\begin{array}{l}0.4056 \\
(0.0090)\end{array}$ & $\begin{array}{l}1.2421 \\
(0.0276)\end{array}$ & $2,660.0479$ \\
\hline & 12-month & $\begin{array}{l}0.9268 \\
(0.4033)\end{array}$ & $\begin{array}{l}-1.2880 \\
(0.5447)\end{array}$ & $\begin{array}{l}0.3626 \\
(0.0079)\end{array}$ & $\begin{array}{l}1.3223 \\
(0.0352)\end{array}$ & $2,461.9173$ \\
\hline
\end{tabular}

Notes. At MLE maximization, data are from daily closing rates for the period from 1st Jan., 2008 to 31st Dec., 2007, total 1,082 data sample; standard deviations are shown in parentheses.

\section{Results and Conclusions}

The inadequacy of the non-satisfactory results obtained from the full period analysis for the period from 1st Jan., 2005 to 22nd Feb., 2012 is due to the following reasons.

As shown in Figure 1 for the period from 2005 to early 2007, the economy of Hong Kong had been relatively stable with pretty low volatilities in interest rate, thus the value of $\gamma>-1$ for all interest rate terms indicating that volatility of interest rate is not sensitive to the level of interest rate, this is consistent with the facts. However, starting from 2008 to beginning of 2012, the Hong Kong and the world economies had been steering from relatively stable to fluctuations, the 2008 financial tsunami initiated by the U.S. had been causing financial instability globally. With the U.S. government taking quantitative easing monetary policy, interest rates in U.S. and Hong Kong fluctuated rapidly from 2008 to beginning of 2012. In short, during the period of early 2008 to early 2012, the value of $\gamma>1$ indicates that volatility of short-term interest rate was extremely 
sensitive. Obviously the term structure of interest rate for these two periods had made major shift, combining the two periods which would lead to unfavorable econometric results.

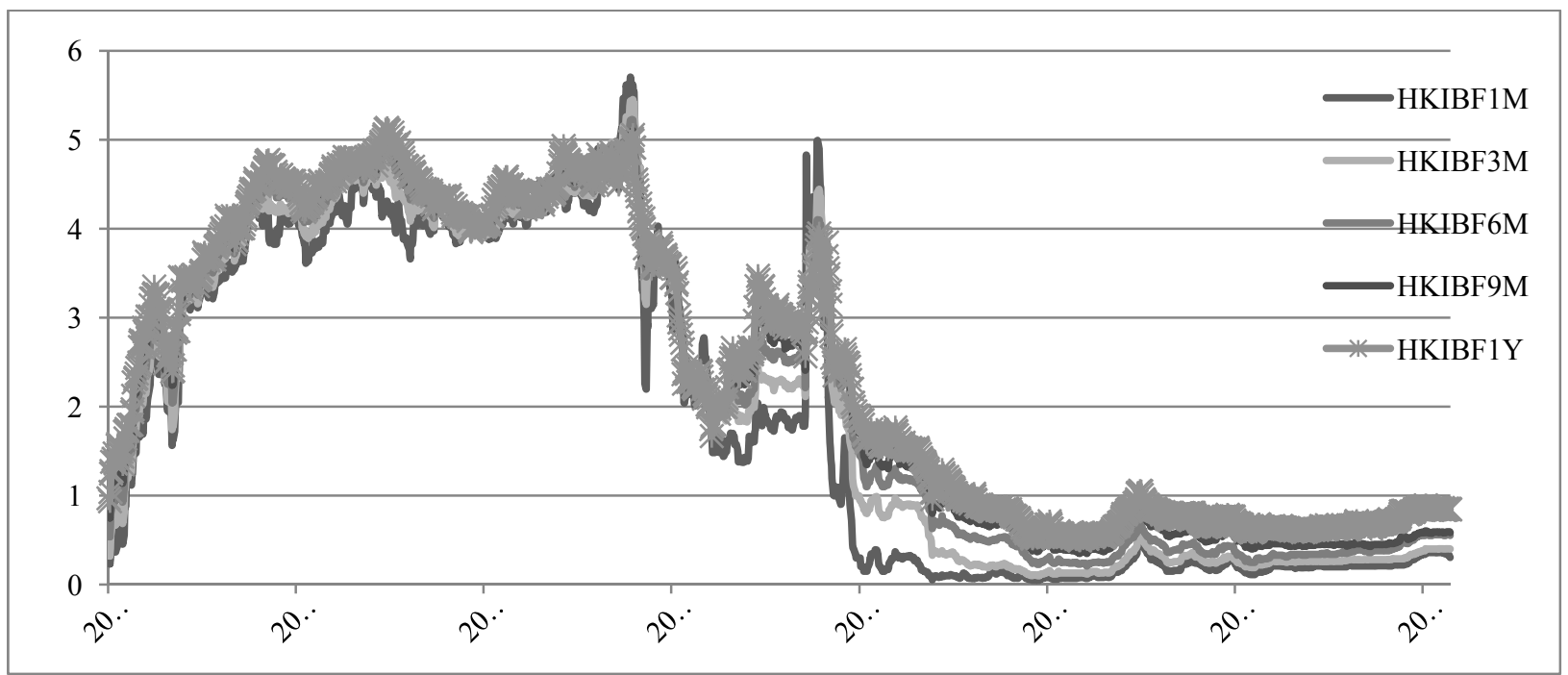

Figure 1. Hong Kong Inter-Bank Offered Rates (HIBOR) for 1-, 3-, 6-, 9-, and 12-month. Note. Data from datastream of Thomson Reuters, 1st Jan., 2005 to 22nd Feb., 2012.

In Table 2, the values of $\beta$ in all nine models are negative (for 1-, 3-, 6-, 9-, and 12-month) and values of $\alpha$ are positive (for 1-, 3-, 6-, 9-, and 12-month), therefore interest rates have mean-reverting nature (for 1-, 3-, 6-, 9-, and 12-month). All parameter vectors ( $\alpha$ and $\beta$ ) are significant, all of them can pass the 5\% significant level. Having $\gamma>-1$, it reveals that volatilities of short-term interest rate are not sensitive to interest rate levels, while $\sigma$ does not equal to zero and $\sigma>0$. It is favorable that parameter vectors $(\gamma$ and $\sigma)$ are significant and all of them can pass the 5\% significant level. The result of model nine (Chan et al., 1992) gives the maximum MLE value: Its effect is more significant than other models.

In Table 3, the values of $\beta$ in all nine models are negative (for 1-, 3-, 6-, 9-, and 12-month), and values of $\alpha$ are positive (for 1-, 3-, 6-, 9-, and 12-month), therefore, interest rates have mean-reverting nature. All parameter vectors $(\alpha$ and $\beta$ ) are significant and all of them can pass the $5 \%$ significant level. Having $\gamma>1$, it reveals that volatilities of short-term interest rate are over-sensitive to interest rate levels, while $\sigma$ does not equal to zero and $\sigma>0$. It is favorable that parameter vectors $(\gamma$ and $\sigma)$ are significant and all of them can pass the 5\% significant level. Again, the result of model nine (Chan et al., 1992) gives the maximum MLE value: Its effect is more significant than other models.

\section{References}

Black, F., \& Scholes, M. (1973). The pricing of options and corporate liabilities. Journal of Financial Economics, 81, 637-654.

Bliss, R. R., \& Smith, D. C. (1994). The elasticity of interest rate volatility. Journal of Financial and Quantitative Analysis, 12 , 65-97.

Brennan, M. J., \& Schwartz, E. S. (1979). Conditional predictions of bond prices and returns. The Journal of Finance, 35, 405-417.

Cai, L., \& Swanson, N. R. (2011). An empirical assessment of spot rate model stability. Journal of Empirical Finance, 18, 743-764.

Chan, K. C., Karolyi, A., Longstaff, F. A., \& Sanders, A. B. (1992). An empirical comparison of alternative models of the short-term interest rate. The Journal of Finance, 47, 1209-1227. 
Corradi, V., \& Swanson, N. R. (2011). Predictive density construction and accuracy testing with multiple possibly misspecified diffusion models. Journal of Econometrics, 161, 304-324.

Cox, J. C., \& Ross, S. A. (1976). The valuation of options for alternative stochastic processes. Journal of Financial Economics, 3 , 145-166.

Cox, J. C., Ingersoll, J. E., \& Ross, S. A. (1985). A theory of the term structure of interest rates. Econometrica, 53, 385-407.

Dahlquist, M. (1996). On alternative interest rate processes. Journal of Banking \& Finance, 20, 1993-1119.

Dothan, L. U. (1978). On the term structure of interest. Journal of Finance, 6, 59-69.

Duong, D., \& Swanson, N. R. (2011). Volatility in discrete and continuous time models: A survey with new evidence on large and small jumps. Advances in Econometrics, 27B, 179-233.

Hiraki, T., \& Takezawa, N. (1997). How sensitive is short-term Japanese interest rate volatility to the level of the interest rate? Economics Letters, 56(3), 325-332.

Manus, D. M., \& Watt, D. (1999). Estimating one-factor models of short-term interest rates (Bank of Canada working paper, pp. 99-118).

Merton, R. C. (1973). Theory of rational option pricing. Bell Journal of Economics and Management Science, 4, 141-183.

Nowman, K. B. (1997). Gaussian estimation of single-factor continuous time models of the term structure of interest rates. The Journal of Finance, 52, 1695-1706.

Nowman, K. B. (1998). Continuous-time short term interest rate models. Applied Financial Economics, 8, 401-407.

Tse, Y. K. (1995). Some international evidence on the stochastic behavior of interest rates. International Money Finance, 14, $721-738$

Vasicek, O. (1977). An equilibrium characterization of the term structure. Journal of Financial Economics, 5, 177-188. 